Chirurg 2019 90 (Suppl 2):S71 https://doi.org/10.1007/s00104-019-0877-5 Online publiziert: 13. Februar 2019

(c) Springer Medizin Verlag GmbH, ein Teil von Springer Nature 2019

\section{Originalpublikation}

Acosta LF, Garcia CR, Dugan A et al (2017) Impact of super obesity on perioperative outcomes after hepatectomy: The weight of the risk. Surgery 162(5):1026-1031

Hintergrund. Mehr als zwei Drittel aller erwachsenen US-Bürger sind übergewichtig bis fettleibig. Seit 1986 hat sich der Anteil der Patienten mit extremer Fettleibigkeit (Body-Mass-Index [BMI] $>50 \mathrm{~kg} / \mathrm{m}^{2}$ ) sogar verzehnfacht. Während ein leicht- bis mittelgradiges Übergewicht einen protektiven Einfluss auf die perioperative Mortalität zeigt, ist bei vielen Eingriffen ein $\mathrm{BMI}>50 \mathrm{~kg} / \mathrm{m}^{2}$ mit einer erhöhten Rate an peri- und postoperativer Morbidität und Mortalität assoziiert.

Der Einfluss extremer Fettleibigkeit auf das postoperative Ergebnis bei Leberteilresektionen ist bisher noch nicht hinreichend untersucht und war Gegenstand der vorliegenden Arbeit.

Methodik. Es wurden alle Patienten von mehr als 600 US-Kliniken betrachtet, die einen BMI $>50 \mathrm{~kg} / \mathrm{m}^{2}$ hatten und zwischen 2005 und 2015 leberteilreseziert wurden.

Ergebnisse. Insgesamt erfolgten 21.228 Leberteilresektionen; davon waren 7271 Patienten übergewichtig; 146 Patienten hatten einen $\mathrm{BMI}>50 \mathrm{~kg} / \mathrm{m}^{2} .72,6 \%$ der extrem Fettleibigen waren Frauen von durchschnittlich 50,6 Jahren. 10,3\% der

Dieser Beitrag wurde erstpubliziert in Der Chirurg (2018) 89:242-242. https://doi.org/10. 1007/s00104-018-0610-9

L. Oczko - F. Rauchfuß - U. Settmacher

Klinik für Allgemein-, Viszeral- und Gefäßchirurgie, Universitätsklinikum Jena, Jena, Deutschland

\title{
Einfluss extremer Fettleibigkeit auf die Morbidität nach Leberteilresektion
}

Patienten wurden einer ASA(American Society of Anesthesiologists)-Klasse $\geq 3$ zugeordnet. Die Mehrheit der Patienten hatte einen behandlungsbedürftigen Hypertonus (69,2\%), Diabetes mellitus (38,4\%) oder Dyspnoe (17,8\%).

Die Operationszeit extrem Fettleibiger war mit durchschnittlich $248 \mathrm{~min}$ deutlich länger als in jeder anderen Gewichtsklasse. Keine der Operationen (Leberteilresektion [74\%], Hemihepatektomie rechts [12,3\%], Hemihepatektomie links [8,9\%], Trisektorektomie [4,8\%]) erfolgte laparoskopisch. Bei $22 \%$ war eine intraoperative Transfusion erforderlich.

Die perioperative Morbidität verdoppelte sich bei extrem fettleibigen Patienten. Ein BMI $>50 \mathrm{~kg} / \mathrm{m}^{2}$ stellte mit $86 \%$ den größten Prädiktor für ein erhöhtes Risiko für infektiöse Komplikationen und mit $63 \%$ einen führenden Einflussfaktor für ein erhöhtes Risiko an Komplikationen, die intensivmedizinische Pflege erforderten, dar. Neben dem BMI $>50 \mathrm{~kg} / \mathrm{m}^{2}$ waren eine ASA-Klasse $>3$, Dyspnoe, präoperativer Gewichtsverlust $>10 \%$, Aszites, ein Hämatokrit $<30 \%$, Nikotinabusus und eine Thrombozytose bedeutende Einflussfaktoren.

Fazit. Extreme Fettleibigkeit, assoziiert mit Komorbiditäten wie Hypertonus, Diabetes mellitus, koronarer Herzkrankheit, Schlaganfall oder Herzinsuffizienz, erhöht das Risiko für perioperative Komplikationen deutlich. Ein BMI $>50 \mathrm{~kg} / \mathrm{m}^{2}$ geht außerdem mit einem gehäuften Auftreten von Leberzirrhose, nichtalkoholischer Steatohepatitis (NASH) und nichtalkoholischer Leberverfettung einher, wodurch das Risiko perioperativer
Morbidität und Mortalität nach leberchirurgischen Eingriffen nochmals erhöht ist. Neben dem Parenchymverlust durch die Leberteilresektion ( $\geq 4$ Segmente), ASA-Klasse $>3$, präoperativem Aszites oder Hämatokrit $<30 \%$ stellt der BMI $>50 \mathrm{~kg} / \mathrm{m}^{2}$ den bedeutendsten Einflussfaktor auf die perioperative Morbidität dar.

Extreme Fettleibigkeit ist mit längeren Operationszeiten, einem längeren Krankenhausaufenthalt, erhöhter Morbidität und einem höheren Ressourcenverbrauch assoziiert. Das Risiko für postoperative Infektionen oder aufwendigere Intensivpflege ist bei diesen Patienten besonders hoch. Ein signifikanter Einfluss extremer Fettleibigkeit auf die perioperative Mortalität im Vergleich zu allen anderen Gewichtsklassen zeigt sich jedoch nicht.

\section{Korrespondenzadresse}

\section{Oczko}

Klinik für Allgemein-, Viszeral- und Gefäßchirurgie, Universitätsklinikum Jena Am Klinikum 1, 07747 Jena, Deutschland Luisa.Oczko@med.uni-jena.de

Interessenkonflikt. L. Oczko, F. Rauchfuß und U. Settmachergeben an, dass kein Interessenkonflikt besteht. 